\title{
Emulation of Ventricular Assist Devices based on Fuzzy Logic Control
}

\author{
B. Jhanvitha ${ }^{1}$, K.S.V. Phani Kumar ${ }^{2}$, and Dr. S. Venkateshwarlu ${ }^{3}$ \\ ${ }^{1}$ PG Scholar, CVR College of Engineering/EEE Department, Hyderabad, India \\ Email: bathula.jhanvitha@gmail.com \\ ${ }^{2}$ Asst. Professor, CVR College of Engineering/EEE Department, Hyderabad, India \\ Email: phani5016@gmail.com \\ ${ }^{3}$ Professor, CVR College of Engineering/EEE Department, Hyderabad, India \\ Email: svip123@gmail.com
}

\begin{abstract}
These days many people are suffering from different heart diseases. These heart diseases or heart failures due to some problems are one of the major reasons for the deaths of many people. To avoid these problems, they are searching for heart donors to transplant the heart. Due to the less availability of heart donors, many people are dying because of heart failure. To overcome this problem, a device needs to be developed that will support the circulatory system of the body. These devices are termed Ventricular Assist Devices (VADs). These VADs are used to help in pumping the blood from the heart to other body parts in case of heart failure. They play a key role in saving the lives of the people who are suffering from different heart diseases and the people who are at the end stage of their heart failure. In previous cases, many of the experiments were conducted on continuous flow systems. But due to some complications that are resulted during their operation these continuous flow devices are not recommended. These continuous flow devices are replaced with pulsatile flow devices which represent the flow of blood from the heart to other body parts. This pulsatile flow can be achieved by controlling the speed of the BLDC motor. To control the speed of the BLDC motor, different controllers such as PID controller and Fuzzy Logic Controllers are used efficiently, which give better results when compared to $P$ and PI controllers.
\end{abstract}

Index Terms: Ventricular Assist Devices; Pulsatile Flow; BLDC Motor; Fuzzy Logic Controller.

\section{INTRODUCTION}

Cardiovascular diseases are the major reasons for death all over the world. Due to fewer heart donors, Artificial Hearts $(\mathrm{AH})$ are the only treatment given to cardiovascular patients. Artificial Hearts starts with design to its implantation to the human body. Artificial Hearts should pass several clinical tests that involve various redesigns and optimizations. During this process, the weight, the dimensions, and the required Electro-Magnetic Forces of the Artificial Hearts are made with perfection. In the medical terminology, there are two types of Artificial Hearts are Total Artificial Hearts and Ventricular Assist Devices. Total Artificial Hearts replaces the human heart. Ventricular Assist Devices supports the human heart. Ventricular Assist Devices allows two therapeutic strategies for heart diseases, which are called bridge to recovery and bridge to transplant. In the first strategy, the heart recovers due to the support of the Ventricular Assist Devices, and in the second strategy, it ensures the patient's survival until a donor or total artificial heart can be transplanted.

\section{A. History and Background}

First, in the inception of the heart program in 1964, investigations in the field have been to develop both Total Artificial Hearts and Ventricular Assist Devices that are implantable without penetrating the skin. To meet this goal, the blood pump, electrical motor, power supply, and volume compensator should be small to fit in the human body and sufficiently durable to support the patient for a long duration. The greatest design challenges have been designed and developed of a reliable, long-lasting, internal electric supply and means of volume compensation as sac type blood pumps eject blood, an equivalent air or fluid must enter into the non-blood side of pump house which prevents a vacuum from forming. Therefore, the development of the volume compensator and the energy source for the pump motor was interdependent. The early method for volume compensation was a compliance chamber which had a variable volume of air in communication and a housing pump. The compliance chamber was a flexible sac which was placed in the chest that would allow a volume of air equal to the stock volume which makes to enter the nonblood chamber of the pump as blood was ejected. Although the compliance chamber initially worked properly within the laboratory, air losses by diffusion and development of animal tissues around the sac eventually rendered the device ineffective.

In addition to this, anticipation is made which makes the change in altitude that would affect air volume in the compliance chamber. Making air travel is impossible for the patients, to resolve this problem of the compliance chamber there is an urgent need for clinical devices Total Artificial Hearts and Ventricular Assist Devices were designed. These devices would have either direct communication with an external pneumatic drive console or an atmospheric vent, which will overcome the need for a compliance chamber. The skin has been penetrated for volume compensation, because of this reason it seemed to be more efficient to transmit electrical power through wires incorporated into the venting apparatus. To overcome the problems related to the volume compensation researchers continued to develop a method that delivers a sufficient amount of electricity to pump the blood. Total Artificial Hearts and Ventricular Assist Devices are electrically driven devices which require a continuous power supply of 8 to $12 \mathrm{~W}$. Conventional batteries were not capable to provide this much amount of power for more than 30 minutes and a nuclear power supply was determined to be impartial and potentially dangerous. 
Therefore, methods for transcutaneous energy transmission were developed ${ }^{[1]}$.

\section{COMPONENTS USED IN VENTRICULAR ASSIST DEVICES}

A ventricular assist device pumps the blood from the left apex into the aorta and could be helpful in $80 \%$ of cases. Because of the risk of infection, a heart assist device has to be implantable. Therefore, we require a highly durable and reliable system which is having the smallest possible volume and weight. The left ventricular heart assist system consists of a blood pump, a centrifugal pump, a brushless D.C. motor, a solenoid, power supply, and controls.

\section{A. Brushless D.C Motors used in Ventricular Assist Devices}

These are electronically commuted dc motors that don't have any brushes. The rotor of a BLDC motor is a permanent magnet but is fixed in place on the stator and the stator has a coil arrangement. These windings can be arranged by either star or delta connections. The stator must be chosen with correct ratings of voltage depending on power supply capability. As the coils do not move there is no need for brushes. Here permanent magnet rotates; the rotation is achieved by changing the direction of magnetic fields generated by surrounding stationary coils. To control the rotation, we adjust the magnitude and direction of current in the rest of the two coils.

BLDC motor works on a similar principle as conventional motors i.e., the Lorentz force law. As a result of reaction forces magnet experiences equal and opposite forces. In BLDC motors current-carrying conductor is stationary while the permanent magnet rotates. When stator coils are switched by a power supply, it becomes an electromagnet and starts producing uniform fields in the area. Due to the force of interaction between electromagnet stator and permanent magnet rotor rotates continuously. The motor produces torque because the development of attraction forces and repulsion forces the motor to move in a clockwise direction. By applying DC power to the coil, the coil will get energized and become an electromagnet. Before energizing a particular stator-winding rotor position acknowledgment is necessary. Hall Effect sensors embedded in the stator sense the rotor position. Therefore, we require a highly durable and reliable system which is having the smallest possible volume and weight. Based on the signals from the sensor controller decides which coil to energize. Low and high-level signals are generated by Hall Effect sensors whenever rotor poles pass near it. The main disadvantage of this motor is that it requires mechanical arrangements to be mounted. Moreover, these sensors are temperature sensitive, due to which the operation of the motor is limited. The reliability of the system is reduced due to excess components and wiring of the system.

1. BLDC without Hall-effect sensors: BLDC motors without the usage of sensors have been used in a larger amount due to their various advantages. Due to the absence of Hall Effect sensors, the action performed by this is obtained from other processes.
a. Back EMF
b. Detecting the conducting state of the diode
c. Back EMF integration

\section{d. Detecting the harmonic voltage}

From the above-mentioned methods, the detection of back EMF method is used widely. The winding's present in the motor helps in creating EMF by using Faraday's Law of Electro 'Magnetic Induction. According to Lenz's law, EMF produced gives rise to a secondary magnetic field that opposes the original change in magnetic flux which helps in the running of the motor. In simpler terms, the EMF resists back EMF. Produced EMF is proportional to the angular velocity of the rotor.

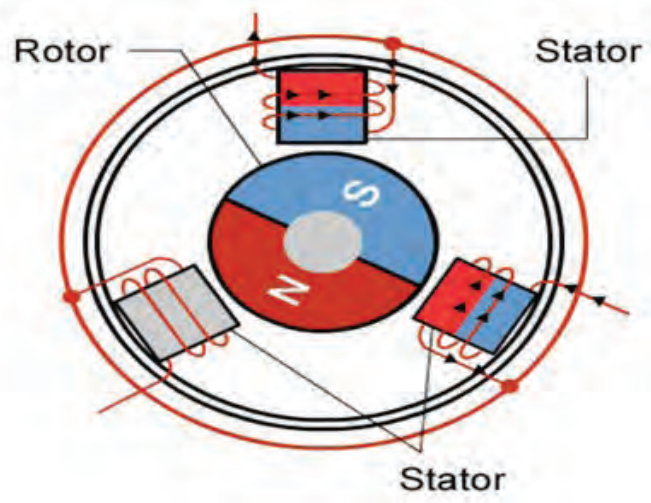

Figure 1. Brushless DC Motor

2. BLDC motors in Ventricular Assist Devices: The range of power in ventricular assist devices is $15 \mathrm{~W}$ to $20 \mathrm{~W}$. The motor which is used should pump $8 \mathrm{~L} / \mathrm{min}$ to $10 \mathrm{~L} / \mathrm{min}$. The hearts have to pump a minimum of $3 \mathrm{~L} / \mathrm{min}$ for a bedridden patient for their survival. Pulsatile flow can be achieved using constant torque and variable systems with motor-pump in ventricular assist devices. For this application, we use Brush-Less DC motors. Generally, a mechanical circulatory support system requires low volume motor pump. BLDC motor is more preferred because of its high efficiency, reliability, smaller volume, and high torque capability ${ }^{[2]}$.

\section{B. Batteries used in Ventricular Assist Devices}

The rechargeable battery pack used for Ventricular Assist Devices should be as small and light as possible for its easy and effective operation. The use of rechargeable lithium cells in implantable medical devices may yield batteries that are smaller and lighter than those of $\mathrm{Ni} / \mathrm{Cd}$ batteries; however, $\mathrm{Ni} / \mathrm{Cd}$ cells are preferred over lithium cells because of their high discharge cycle and life cycle. The following are the corresponding characteristics of rechargeable batteries. Testing done on various batteries is shown in the figure below which is varying at a temperature of 45 degrees ${ }^{[3]}$.

Various batteries were considered for supplying power to Ventricular assist devices starting from percutaneous lead cables to a Free-range Resonant Electrical Energy-Delivery (FREE-D) system. Where all of them have both advantages and disadvantages. Some of the new batteries charging systems have been evolving day by day. Such as Solar based recharging systems, Proton exchange membrane fuel cells, and Skeletal muscle energy. The evolution has been started from using battery technology to the above discussed. These emerging technologies hold promise for increasing the life 
of the battery. Out of these FREE-D systems holds good for delivering power to ventricular assist devices.

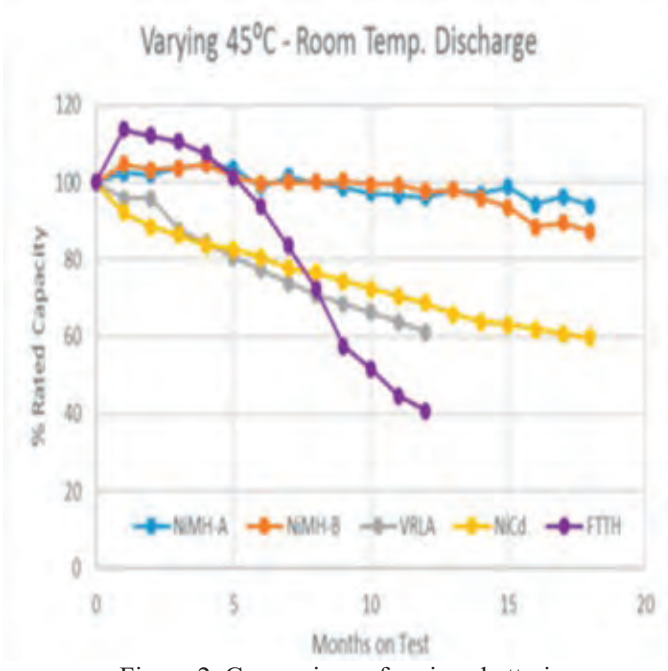

Figure 2. Comparison of various batteries

Wireless power to Ventricular assist devices is provided by FREE-D systems which make use of strong resonant coupling technology. FREE-D system achieves high power transfer efficiency up to $90 \%$ for nearly any angular orientation over a large of separation distances. They are two types of power circuits. FREE-D technology could be applied in other medical applications that require implanted batteries, such as pacemakers, implanted defibrillators, cochlear implants. Outside medicine, FREE-D technology is applied in some ideal consumer electronic applications mobile charging, electric car charging, and kitchen appliance operation. Future work for this FREE-D systems to power VADs a full characterization of relay resonators presented and the resonators to improve the system efficiency and enable single-frequency operation.

\section{Pumps used in Ventricular Assist Devices}

In past decades therapeutic instruments have greater innovation in them and have been widely used for the treatment of cardiac arrest. In general, these instruments consist of different types of blood pumps with different pumping principles ranging from intra-aortic balloon pumps to total artificial heart (TAH). In fact, in recent years there has been more Left Ventricular Assist Devices (LVAD) than heart transplants. These devices not only sustain life but also improve the quality of life of patients with end-stage heart failure. Total Artificial Hearts usually follow Left Ventricular Assist Devices by a certain lag because it applies to fewer people.

All currently used clinically are total artificial hearts which are pulsatile. There is continuous improvement in certain areas for more physiological application of it. One of the most common adverse effects of Left Ventricular Assist Devices use is the development of right heart failure. As many as $30 \%$ of Left Ventricular Assist Devices patients exhibit some degree of right heart failure that requires prolonged in tropic support. Hence there is a need for a bi- ventricular assist device has arisen which needs a total artificial heart.

Ventricular Assist Devices includes blood pumps for its operation. In general blood pumps are categorized into two types, they are displacement pumps and rotatory pumps in which displacement pumps are used for clinic and medical purposes $^{[4]}$.

At present rotatory pumps which includes centrifugal pump has been widely used which works on the principle of energy transferred at larger volume with low pressure. The main advantage of this pump is better transportability, less spallation, and lower blood damage for cardiopulmonary bypass.

\section{Controllers used in Ventricular Assist Devices}

For controlling the speed of the brushless DC motors various controllers are used nowadays. They are termed as Proportional Integral (PI), Proportional Integral Derivative (PID), Fuzzy Logic Controller (FLC), and various combinations between them. Out of various controllers, the Fuzzy Logic Controller serves as a solution for highperformance electric drives.

Due to various disadvantages of PI Controllers such as slow response, sudden change in load torque and sensitivity to gains of the controller. Because of such reasons, there has been an increasing demand for non-linear control structures such as Fuzzy Logic Controllers which are inherently robust to disturbances caused due to loads. Due to variations in load disturbances and parameters, BLDC motors being nonlinear are greatly affected.

Fuzzy Logic Controllers can model non-linear systems very effectively. It makes use of linguistic variables for the operations required. It is a very easy and feasible method for defining the characteristics of a nonlinear system during implementation and designing. Reference value and speed error value are the two main factors considered for comparing the speed of the motor. The main components of the Fuzzy Logic Controller are fuzzification, fuzzy inference defuzzification.

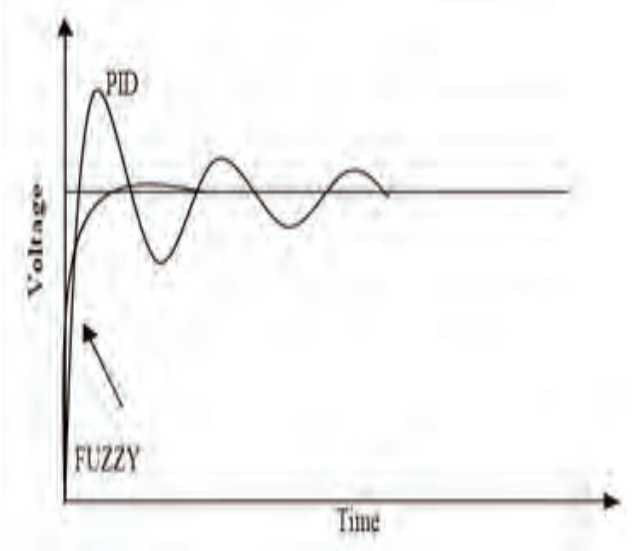

Figure 3. The response of the fuzzy controller 


\section{EXPERIMENTAL EVALUATION OF VENTRICULAR}

\section{ASSIST DEVICES}

Based on the results of optimization, the prototype was constructed. The blood chamber and valves are modified. This made pumping capacity to increase from $50 \mathrm{ml}$ to $60 \mathrm{ml}^{[5]}$.In addition to this wear, prone components such as fragile coupled gear and the ball bearings have been replaced by linear moving part, the magnetic attraction forces between stator and rotor are avoided minimizing the current supplying springs as healed a duration for more than 5 years.

\section{A. Working of Ventricular Assist Devices}

Blood is continuously drained from the Left Ventricular chamber via the apical inflow canal and propelled through pump housing where the magnetic field generation by a rotary pump transmits blood through the outflow graft anastomosed to the ascending aorta. A percutaneously tunneled driveline connects the external power source or the system controller to the pump. We can observe how ventricular assist devices are connected to the human body. The system controller has both manual and fixed settings that modulate pump speed, provided hazard alarms, log any device malfunction for future analysis. This device delivers 3 to $10 \mathrm{~L}$ per min of flow at pump speeds of 6000 to $15000 \mathrm{rpm}$. The batteries used here to power the device may last $12 \mathrm{hrs}$ before it should be recharged.

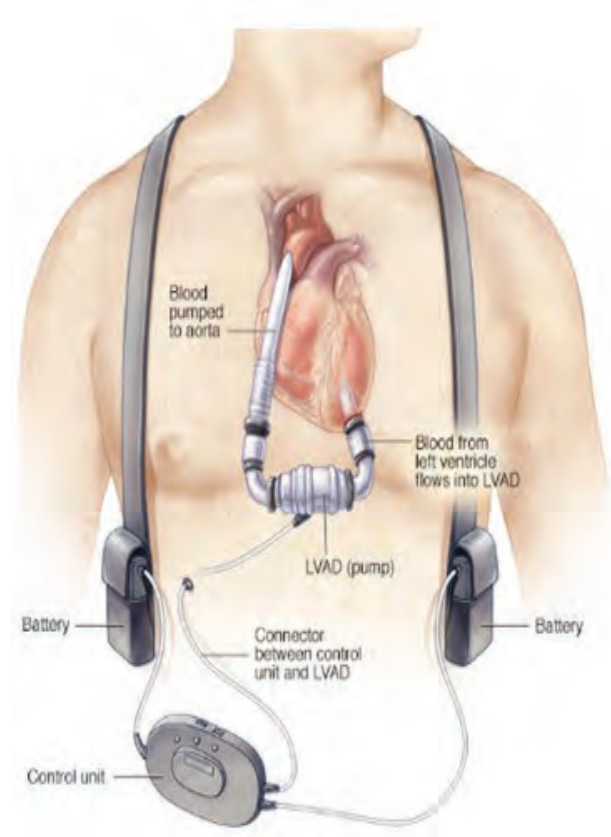

Figure 4. Ventricular assist devices are connected to the human body

Ventricular Assist Device is a centrifugal, continuous flow, implantable rotary pump currently in clinical practice. This third-generation Ventricular Assist Device operates via a hydro-magnetically levitated rotor without mechanical bearings and can deliver up to $10 \mathrm{~L} / \mathrm{min}$. Elimination of the contact bearings imparts several clinically relevant structural, and theoretical advantages relative to the HM 11. Firstly by affording further miniaturization this $140 \mathrm{~g}$ pump can be implanted between the pericardial space, thus simplifying the operative placement by eliminating the need of creating a pump pocket. This miniature size also makes the device more conducive to minimally invasive implantation techniques. The absence of mechanical contact within the pump eliminates friction and heat generation which also improves device durability. Generally, the pump is set at the range of 2400 to $3200 \mathrm{rpm}$ to deliver flow rates between 3 and $8 \mathrm{~L} / \mathrm{min}$. The batteries which power the devices can deliver 4 to $6 \mathrm{~h}$ of support when fully charged.

\section{EMULATION ENVIRONMENT}

Mechanical circulatory support helps to keep their heart pumping enough blood. It acts as a supplement or replacement for the action of the failed heart. Introduction of pulsatility to the motor by varying the speed during rotation. Implementation is done using a microcontroller for the BLDC motor. In this, we are using a BLDC motor which is used to pump the blood. BLDC motor is used because of its advantages such as it as high-speed control during at high voltage and low voltage-time and it has constant torque while changing the speed of the motor.

TABLE I.

REFERENCE INPUT FOR THE SPEED CONTROLLER OF THE MOTOR.

\begin{tabular}{|c|c|c|}
\hline S. No & Speed (rpm) & Time (sec) \\
\hline 1 & 1200 & $0+$ \\
\hline 2 & 1600 & 0.2 \\
\hline 3 & 1400 & 0.35 \\
\hline 4 & 1300 & 0.5 \\
\hline 5 & 1220 & 0.6 \\
\hline 6 & 1200 & 0.7 \\
\hline 7 & 1200 & 1 \\
\hline
\end{tabular}

The power to the BLDC motor is given by a battery which is connected to the controller. Without applying any load on the motor we can control the speed of by motor which varies accordingly by using ATMEGA328p. In this paper, reference speed values of a repeating sequence block are considered which are as follows.

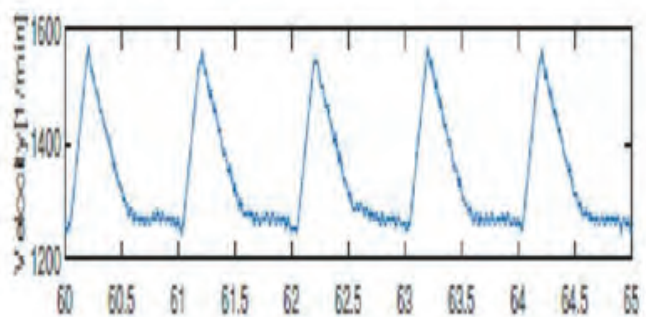

Figure 5. Reference input graph for the speed controller of the motor.

Before controlling the speed of the brushless dc motor at various speeds, we need to find out whether the motor is rotating or not. For this process, we can make use of a proximity sensor which helps in detecting an obstacle which therefore helps in finding total revolutions per minute i.e 
(rpm). Proximity sensors provide medium or low-resolution sensing, depending on the total number of pulses measured per revolution. A bolt attached to the shaft can be sensed using a proximity sensor. Revolutions per minute can be calculated by using the formula (1) given below.

$$
R P M=\text { Pulse Frequency } * 0.1
$$

Whereas the number of pulses is being sensed per revolution is termed as pulse frequency.

\section{A. Algorithm for Obtaining RPM using a Proximity Sensor}

STEP 1: First we need to set and initialize the variables such as proxy, s, Val, prev_state, current_state, count, and formula of rpm.

STEP 2: Initialize current_state as equal to the proxy.

STEP 3: Here we need to check for the condition whether current_state is not equal to prev_state are not.

STEP 4: If this condition is false again we have to go back to STEP 2 .

STEP 5: If the condition is true check whether the obtained current state is equal to one.

STEP 6: If the condition is false, go back to STEP 2.

STEP 7: If the condition is true increment the count value by $1($ count $=$ count +1$)$.

STEP 8: Completing the loop initializes the prev_state value is equal to the current state value.

STEP 9: By using this formula we can calculate the rpm of the motor.

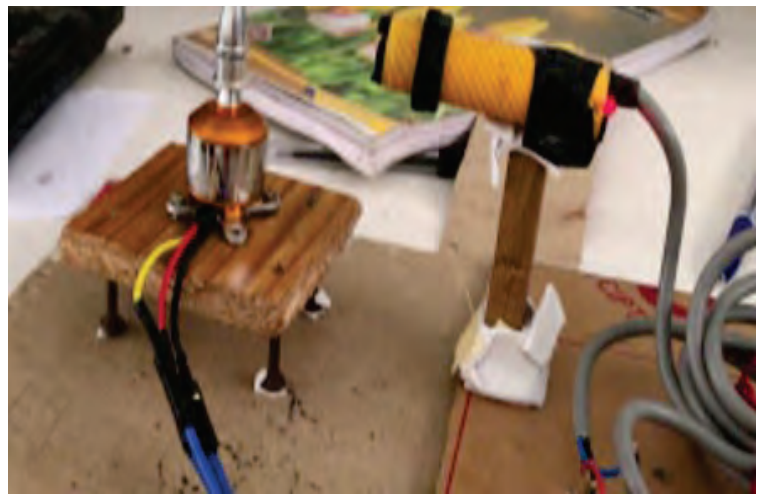

Figure 6. Finding the speed of the BLDC motor using the proximity sensor

This method produces low resolution and it acts as a major drawback. To overcome this we can make use of two bolt heads by which resolution improves but often pulses are inconsistent and they are not symmetrical. Hence we can directly control the speed of the motor by writing a code in the microcontroller.

For achieving the speed values of the above-shown Table. 1 we require a brushless dc motor, ATMEGA328p microcontroller, and a battery. The block diagram for this operation is as shown below. Here the variations in the speed can be obtained by programming ATMEGA328p. The programming is done in such a way that the motor starts running as soon as the battery is connected to the brushless dc motor. ATMEGA328p programming is described in the algorithm accordingly.

\section{B. Block Diagram for Controlling the Speed}

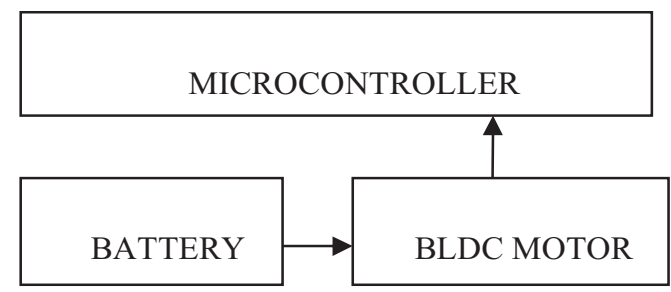

Figure 7. Block diagram for speed control of motor

\section{Algorithm for Controlling the speed of the Motor}

STEP 1: First we have to initialize the values which we are using in this code.

STEP 2: Next step is we have to declare each input and output pin in which tells the Arduino how to configure a certain pin. In this, we also define the serial begin which is 9600 in this program which defines the baud rate from which the program will begin.

STEP 3: The next step is the void loop () where we specify the main behavior of our interactive device (Arduino). This will be repeated over and over again until you remove power from the board.

STEP 4: In this step, we use 6 different types of for loops in which it is described with different speed variations with certain delay time.

STEP 5: In the first for loop we give the different values like from $1200 \mathrm{rpm} 1600 \mathrm{rpm}$ for which the motor rotates at given speeds with an increment of speed for $400 \mathrm{rpm}$ with the delay of 0.2 seconds.

STEP 6: In the second for loop we give the different values like from $1600 \mathrm{rpm}$ to $1400 \mathrm{rpm}$ for which the motor rotates at given speeds with the decrement of speed for 200 rpm with the delay of 0.15 seconds.

STEP 6: In the third for loop we give the different values like from $1400 \mathrm{rpm}$ to $1300 \mathrm{rpm}$ for which the motor rotates at given speeds with the decrement of speed for $100 \mathrm{rpm}$ with the delay of 0.15 seconds.

STEP 7: In the fourth for loop we give the different values like from $1300 \mathrm{rpm}$ to $1220 \mathrm{rpm}$ for which the motor rotates at given speeds with the decrement of speed for 80 rpm with the delay of 0.1 seconds.

STEP 8: In the fifth for loop we give the different values like from $1220 \mathrm{rpm}$ to $1200 \mathrm{rpm}$ for which the motor rotates at given speeds with the decrement of speed for $20 \mathrm{rpm}$ with the delay of 0.1 seconds.

STEP 9: In the last for loop we give the different values like from $1200 \mathrm{rpm}$ to $1200 \mathrm{rpm}$ for which the motor rotates at given speeds with the constant speed with a delay of 0.3 seconds.

STEP 10: After this, the loop will end and we will come out of the loop and the program will stop. 
D. Results for Controlling the Speed of the Motor

After dumping the code into ATMEGA328p and uploading it, we can observe the rotation of the motor. The motor continues to rotate at various speeds accordingly until the loop is completely executed. We can observe the changes in the speed from the serial monitor of the microcontroller.

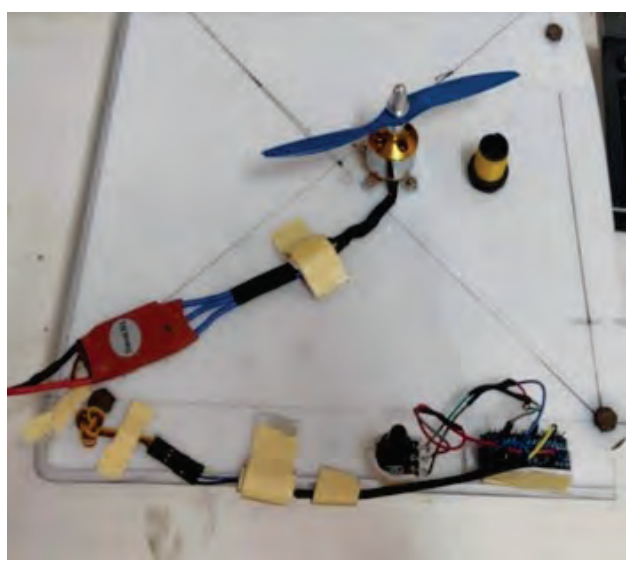

Figure 8. Prototype for controlling the speed after writing a program

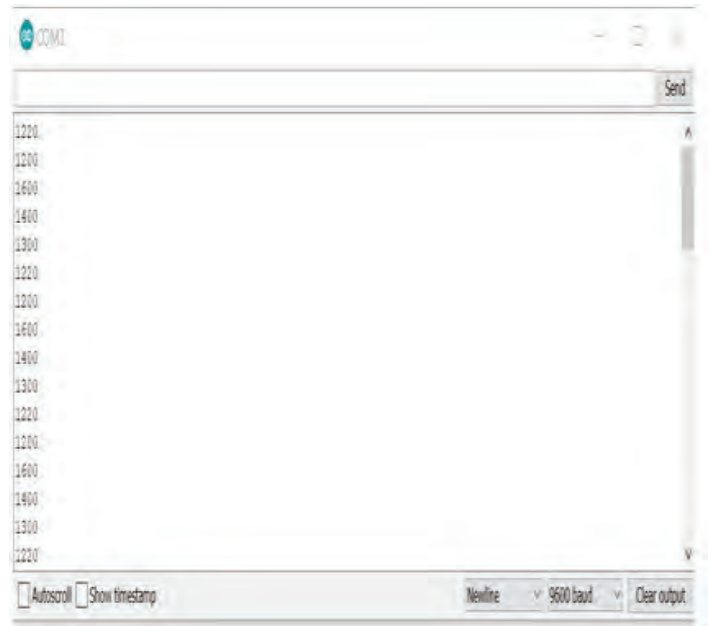

Figure 9. Output values from the serial monitor

TABLE II.

ACTUAL VAlues ObSERVED IN SPEEDS OF THE MOTOR USING THE DigitAL TACHOMETER

\begin{tabular}{|c|c|c|}
\hline S. No & Speed (rpm) & Time (sec) \\
\hline 1 & 1205 & $0+$ \\
\hline 2 & 1602 & 0.2 \\
\hline 3 & 1404 & 0.35 \\
\hline 4 & 1306 & 0.5 \\
\hline 5 & 1228 & 0.6 \\
\hline 6 & 1209 & 0.7 \\
\hline 7 & 1210 & 1 \\
\hline
\end{tabular}

By using this procedure we could emulate the speeds of BLDC Motor to the actual graph. But we could find some errors in the speed achieved by the motor. To overcome these issues Fuzzy Logic Controller can be used. We can detect the speed of the brushless dc motor by making use of a digital tachometer. The speed values for which error has been observed are as follows.

\section{E. Block Diagram using Fuzzy Logic Control for Rectifying the Errors in Speed}

Generally, for using Fuzzy Logic Control we need to calculate the errors and change in error values. Here we come across two speed values one is 'Set Value' which is the reference values considered for the variations in speed using a repeating sequence. Another speed value is termed as the 'Actual Value' which is obtained from the digital tachometer readings. Using set values and actual values we can determine the 'Error' i.e. the difference between the set value and the actual value is termed as the Error.

Error $=$ Set value - Actual value

The difference between two error values at a particular instance of time is termed as a change in error.

Chango in orror $-\frac{\operatorname{Error}(2)-\operatorname{Error}(1)}{t}$

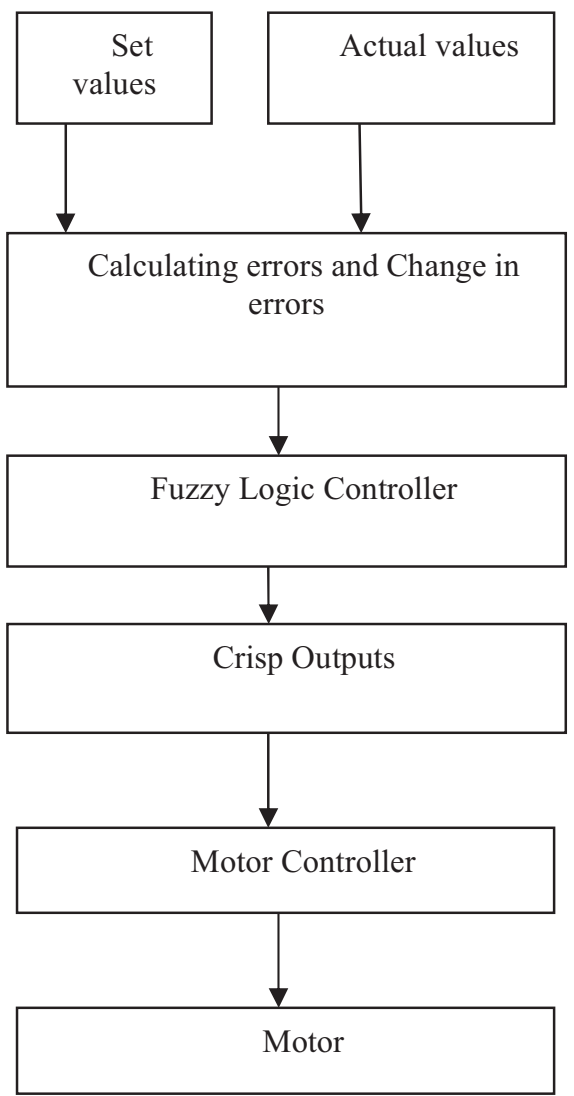

Figure 10. Block diagram for rectifying errors in speed 


\section{F. Flowchart for Fuzzy Logic Controllers for Rectifying the Errors in Speed}

The fuzzy logic controller plays a vital role in the process of finding the error and change in error values until the crisp output is found.

In the process of fuzzification membership functions are applied to the measurement and the degree of membership is determined. For calculating the degree of membership we need to calculate the values of $\delta_{1}$ and $\delta_{2}$. These two values can be computed as follows.

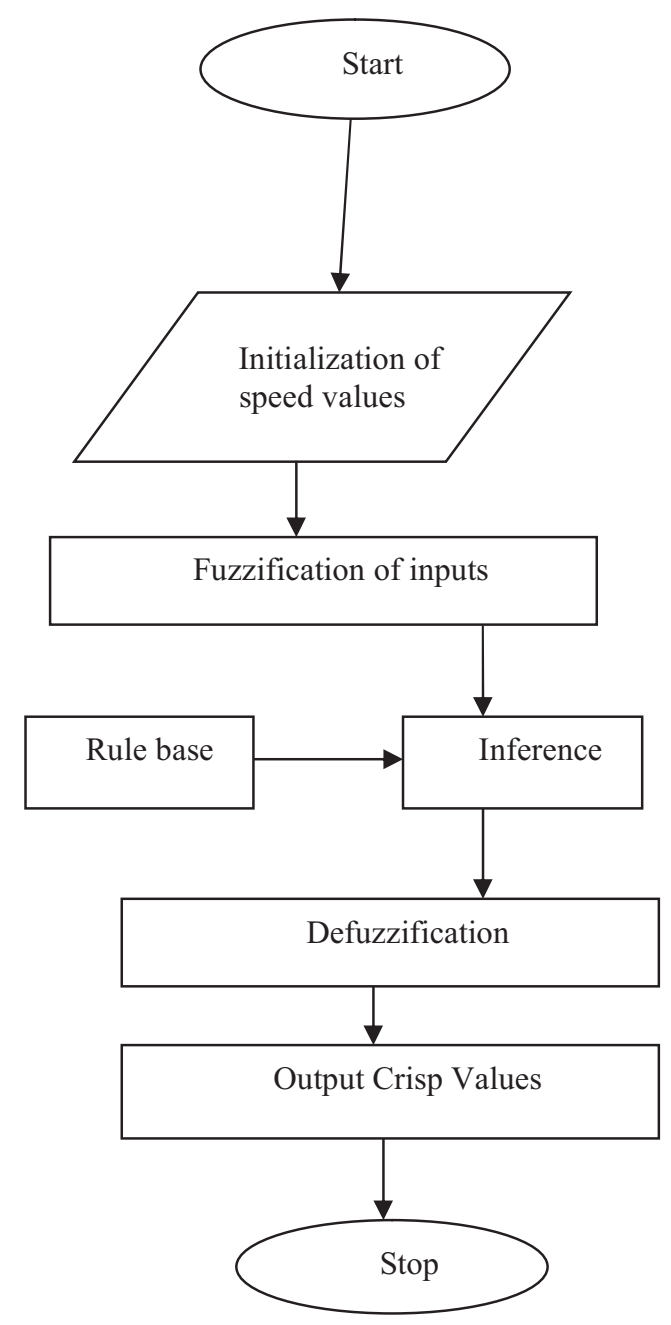

Figure 11. Flowchart for rectifying errors in speed

$$
\begin{aligned}
& \delta 1=x-\text { Point } 1 \\
& \delta 2=\text { Point } 2-x
\end{aligned}
$$

Point 1 and Point 2 are the starting and ending values i.e. first and last limits of the various membership functions. And $\mathrm{x}$ is the input error value. Then from equations (4) and (5), if $\delta_{1}<=0$ and $\delta_{2}<=0$, the degree of membership is considered as zero. In case if anyone of the conditions is not satisfied, we need to compute for the degree of membership in another manner using the slopes of the chosen membership function which is as follows.

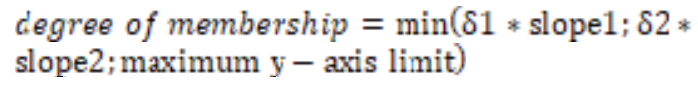

Rule Base contains the fuzzy logic gradation of the expert's linguistic description in the form of a set of rules of how to achieve good control.

Inference engine and fuzzy inference module are the synonyms for "inference mechanism". It helps in emulating the expert's decision in applying and rendering the knowledge which helps in knowing which is best to manage the plant. This helps in evaluating the control rules which are relevant at present time and decides what input to the plant should be.

The defuzzification interface converts the conclusions obtained by the inference mechanism into the actual inputs. Converting decisions into actions is the process of defuzzification. After defuzzification output, crisp values are obtained. Defuzzification can be performed using various methods such as Center of Sums Method; Center of gravity or Centroid of Area Method; Center of Area or Bisector of Area Method; Weighted Average Method and Maxima Method again in Maxima method as First of Maxima Method; Last of Maxima Method; Mean of Maxima Method. Out of which mostly the Center of gravity method and Center of area method are used widely.

For writing a code using Fuzzy Logic Control in the microcontroller we need to follow all the above-said steps accurately. While writing the program we need to initialize the entire variable. We need to consider the rule base according to our requirements. These crisp values are fed to the motor controller and balances the errors produced. The whole process of fuzzification to defuzzification can be done by writing a code in the microcontroller depending on the changes that occurred. By using this procedure, we can eliminate the errors that occurred and emulate them to the actual graph results.

\section{CONCLUSIONS}

By pulsating the speed of the motor, the pulsatile flow of the blood in mechanical circulatory devices can be achieved. According to the dimensions, weight, and simplistic control, a practicable solution is obtained by the BLDC motor. Controlling of BLDC motor's speed can be achieved using various methods as discussed above. The ripples in the speed of the motor while knowing the pulsatility in speed may cause turbulence in blood flow. So, to reduce the ripples in speed we can use various control techniques. Fuzzy-based control results in a smaller number of overall ripples get reduced increasing the performance and smaller percentage of peak overshoot in motor speed than simple PI, PID, and fuzzy, fuzzy PI are obtained by the combination of Fuzzy and PID.

\section{REFERENCES}

[1] Slaughter MS, Myers TJ. "Transcutaneous energy transmission for mechanical circulatory support systems: history, current status, and future prospects". J Card Surg 2010;25:484-9. 
[2] P. Damodharan and Krishna Vasudevan, Member, IEEE on "Sensorless Brush less DC Motor Drive Based on the ZeroCrossing Detection of Back Electromotive Force (EMF) From the Line Voltage Difference" IEEE transactions on energy conversions, VOL. 25, NO. 3, September 2010.

[3] MacLean GK, Aiken PA, Adams WA, Mussiv and T. "Comparison of rechargeable lithium and nickel/cadmium battery cells for implantable circulatory support devices". Artif Organs 1994;18:331-4.

[4] Jan D. Schmitto, MD, Daniel Burkhoff, Murat Avsar, Oliver Fey, Petra Ziehme, Gwen Buechler, Axel Haverich, and Martin Strueber, From the Department of Cardiac, Thoracic, Transplantation, and Vascular Surgery, Hannover Medical School, Hannover, Germany; Department of Cardiology, Columbia University, New York, New York; and CircuLite Inc, Saddle Brook, New Jersey. On "Two axial-flow Synergy MicroPumps as a biventricular assist device in an ovine animal model"; The Journal of Heart and Lung Transplantation, Vol 31, No 11, November 2012.

[5] André Pohlmann, Marc Lebmann, Thomas Finocchiaro, Thomas SchmitzRode_, and Kay Hameyer on "Numerical Computation Can Save Life: FEM Simulations for the Development of Artificial Hearts"; IEEE transactions on magnetics Vol. 47, No. 5, May 2011.

[6] B. Lima, Michael M., Gonzalo V. G. Stawinski. "Ventricular assist devices: The future is now". Elsevier publications on Trends in cardiovascular medicine 25 (2015).

[7] Waters BH, range resonant electrical energy delivery (FREED) system. Proc IEEE 2012;100:138-Sample AP, Bonde P, Smith JR. Powering ventricular assist device (VAD) with the free- 49.

[8] Steven Keeping "Controlling Sensorless, BLDC Motors via BackEMF"2013-06-19.

https://www.digikey.com/en/articles/techzone/2013/jun/contro $\underline{\text { llingsensorless-bldc-motors-via-back-emf }}$ 